\title{
The meteoroid fluence at Mars due to Comet C/2013 A1 (Siding Spring)
}

\author{
Atthea Moorhead \\ NASA Meteoroid Environment Office \\ Collaborators: \\ Paul Wiegert (UWO). \& Bill Cooke. \\ Rhiannon Blaauw \& Aaron Kingery \\ Will Yoder \& Cameron McCarty
}

May 2, 2014 
On October 19, 2014, Comet C/2013 A1. (Siding Spring) will pass within $150,000 \mathrm{~km}$ of Mars.

This is closer than all"known Earth-comet encounters.

A collision has been ruled out, but Mars and its manmade satellites will pass through the coma and tail.

Mars will be showered with meteors and satellites will have an increased risk of meteoroid impacts. 


\section{Outline}

(1) Comet background

- C/2013 A1 (Siding Spring)

- Mars encounter

- Mars spacecraft

(2) Coma model

- Analytic model

- Validation and simulations

- Effects at Mars

(3) Recent observations

- Brightness monitoring

- Observational constraints

- Comparison with Hale-Bopp 


\section{Comet types: classed by orbit}

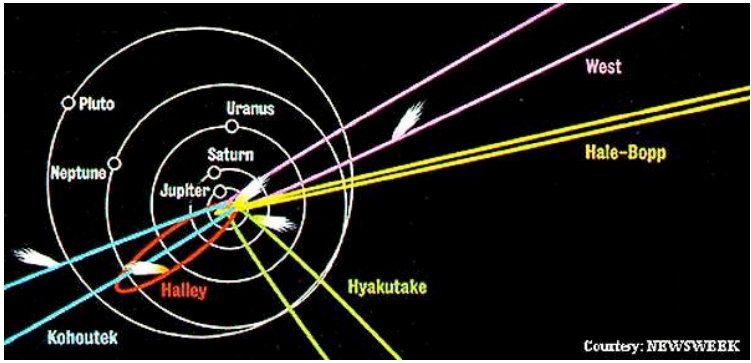

Type

Orbit

Origin

Examples

Short period

$P<200$ yrs

Kuiper Belt Halley

Long period (Oort cloud)

$P \sim 1000$ s yrs $\quad$ Oort cloud

Sungrazers

Pass near or plunge into the Sun

Hale-Bopp,

C/2013 A1

C/2012 S1 (ISON) 


\section{Comet properties}

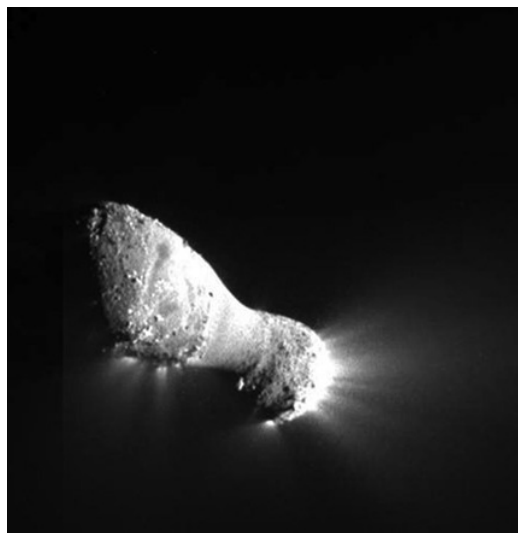

- Comets are the least reflective objects in the Solar System:

- Halley has an albedo of about 4\%.

- Asphalt has a albedo of $7 \%$.

- Long period comets contain more volatiles, as short period comets lose much of these in frequent passages around the Sun. 


\section{Cometary comae}

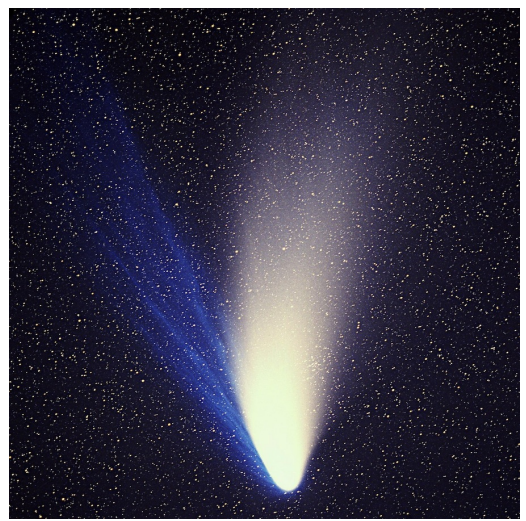

- Hale-Bopp:

- Discovered at 7.2 AU

- Coma was 1 million km in August 1995

- Siding Spring:

- Imaged at $10 \mathrm{AU}$

- Already active

Comet Hale-Bopp, NASA 


\section{C/2013 A1 (Siding Spring)}

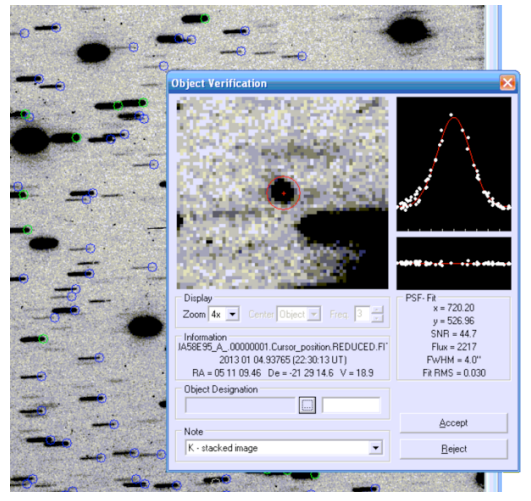

- Discovery:

- January 3, 2013

- Rob McNaught

- 0.5 meter telescope

- Siding Spring, Australia

- Pre-discovery images located in Catalina Sky Survey

- Active in earliest images (Dec. 8, 2012)

- Hyperbolic orbit 


\section{NEAR MISS!}

JAN 3 Comet discovered at

2013 Australia's Siding

Comet Siding Spring (C/2013 A1) is racing toward Mars for a close encounter in October, 2014.

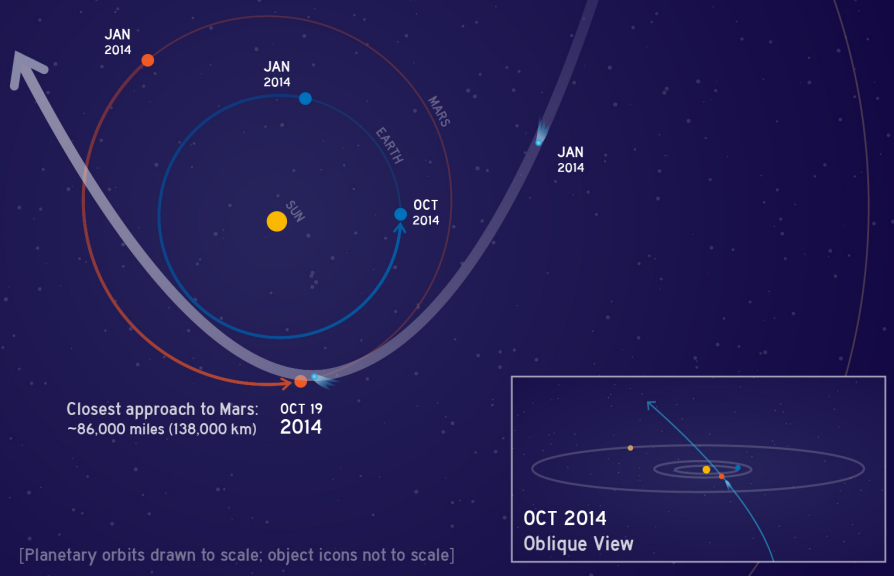




\section{Close encounter with mars}

Movie credit: Leonid Elenin using SpaceEngine software 


\section{Close encounter with mars}

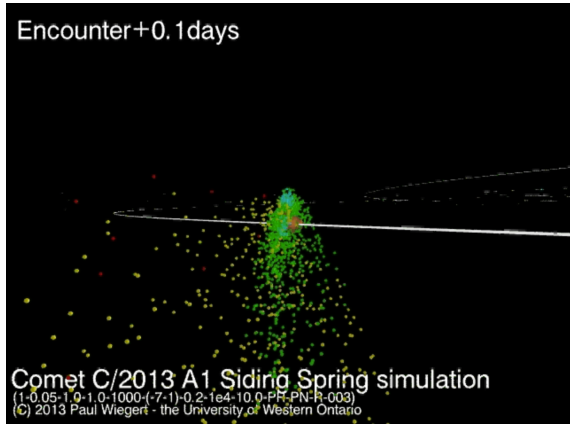

- Comet is north of Mars's orbital plane at closest approach

- Close approach: 131,000 - 145,000 km

- Approach distance nominal value: 138,000 km

- Coma/tail may envelop Mars 


\section{Mars satellites}

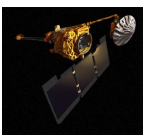

Odyssey (NASA) $400 \mathrm{~km}, 2 \mathrm{hr}$ orbit

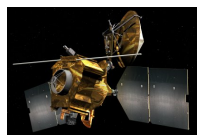

MRO (NASA)

$300 \mathrm{~km}, 2 \mathrm{hr}$ orbit

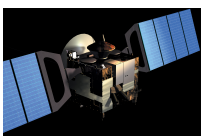

Mars Express (ESA) $300-10,000 \mathrm{~km}$ $7.5 \mathrm{hr}$ orbit

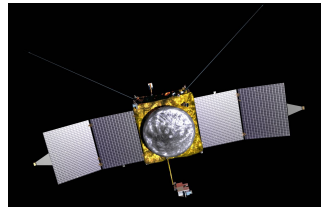

MAVEN (NASA)

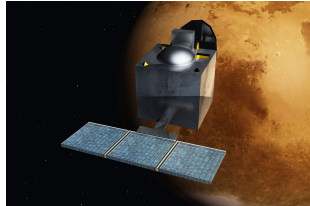

MOM (ISRO)

$150-6,200 \mathrm{~km}, 4.5 \mathrm{hr}$ orbit $365-80,000 \mathrm{~km}, 76.7 \mathrm{hr}$ orbit Arrives September 2014

Arrives September 2014 


\section{Meteoroid impact risks}

- Impacts to critical components

- Sudden attitude changes

- Electrostatic/EMP effects may occur, depending on environment and spacecraft charging state

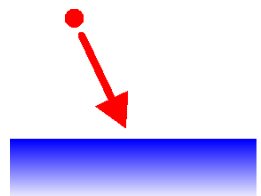

Impact

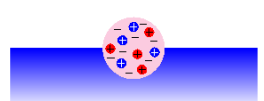

Plasma formation

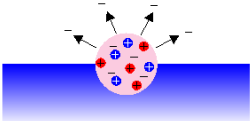

Initial electron motion

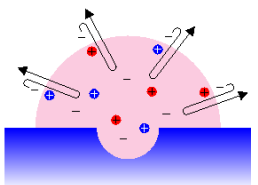

Plasma expansion

Image courtesy of MEDUSSA 


\section{Meteoroid impact risks}

- Encounter speed similar to Perseids at Earth

- Two spacecraft anomalies attributed to Perseids:

- Landsat 5 lost attitude control during 2009 Perseids

- OLYMPUS satellite lost during 1993 Perseids

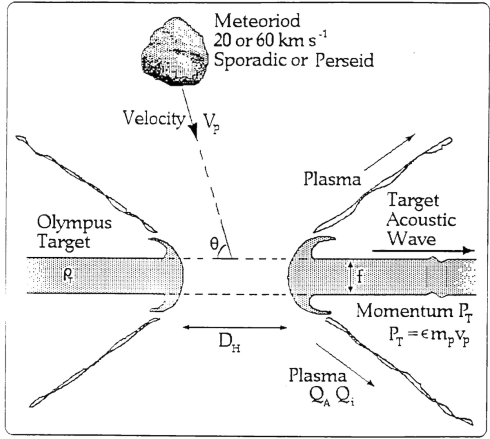

Image from McDonnell et al., 1993 


\section{Possible mitigation strategies}

- Align solar arrays sunward, edge-on to meteor shower

- Sun-Mars-comet angle is $90.2^{\circ}$

- Present hard side to radiant

- Phase orbit to use Mars as a partial shield:

- Depends on orbit geometry

- Shower will last a few hours, dependent on coma size (30 min per 100,000 km of coma/tail)

- Odyssey and MRO have 2 hour orbits 


\section{Particles in the coma and tail of Siding Spring}

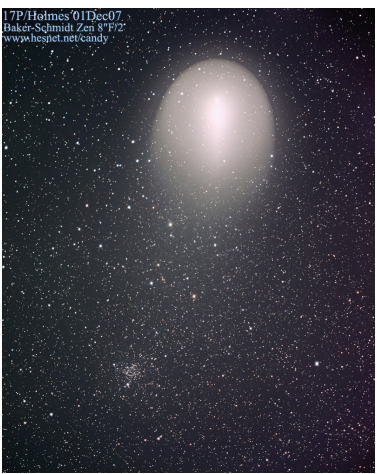

- Coma and tail contains both gas and particles

- Both grow as heliocentric distance decreases

- Fluence - flux integrated over Mars/spacecraft trajectory

- Direct measure of spacecraft risk

- We compute the particle fluence at Mars using properties of Siding Spring (magnitude, orbit), supplementing with Halley data (particle albedo, size distribution) where necessary. 


\section{The Giotto flyby of 1P/Halley}

- We have detailed coma data for one comet: Halley.

- Giotto recorded 12,000 impacts.

- Model fits to these data yield:

- Particle density and albedo

- Particle size distribution

- Particle spatial distribution

- Total number of particles derived from Siding Spring magnitude, not Halley.

HMC 68 Image Composite Comet Halley 14th March 1986 


\section{Analytic model}

Quantifying the number of $\sim 100$ micron $^{1}$ or larger particles in the coma/tail:

- Determine the brightness at the time of the encounter.

(2) Use particle albedo to compute the total particle surface area.

(- Combine with Halley-like particle size distribution and material density to compute number of particles.

( - Use $r^{-2}$ spatial distribution to compute the number density.

(- Integrate along the trajectory to get fluence.

Our analytic model can be used to quickly calculate new fluence estimates as comet properties are measured/constrained.

${ }^{1} 100$ micron particles are capable of cutting exposed spacecraft wires. Mass limit is $4 \times 10^{-6} \mathrm{~g}$, actual size limit depends on density 


\section{Analytic model}

$$
\begin{aligned}
\sigma_{*}=\frac{g h^{-\beta}}{a} & \left(\frac{2}{\pi}\right)^{\frac{1}{3}}\left(\frac{\rho}{3}\right)^{\frac{2}{3}} 10^{-0.4\left(M 1-m_{\odot, 1 a u}\right)} \mathrm{au}^{2} \\
& \times\left(\frac{3-k}{1-k}\right)\left(\frac{m_{\max }^{(1-k) / 3}-m_{*}^{(1-k) / 3}}{m_{\max }^{(3-k) / 3}-m_{\min }^{(3-k) / 3}}\right) \\
& \times \frac{\cos ^{-1}\left(b / r_{c}\right)}{b r_{c}}
\end{aligned}
$$




\section{Analytic model}

Dependence on comet/meteoroid properties
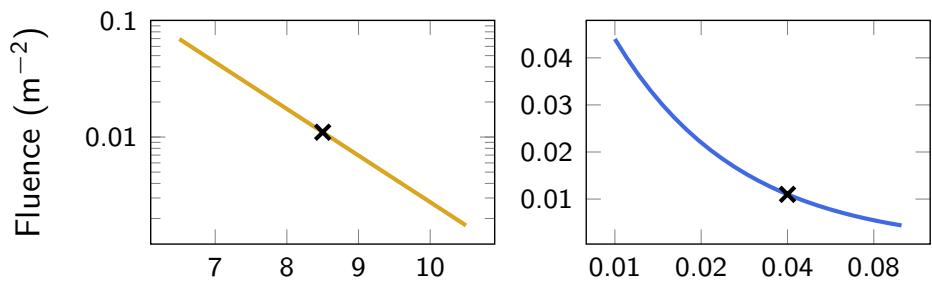

comet magnitude

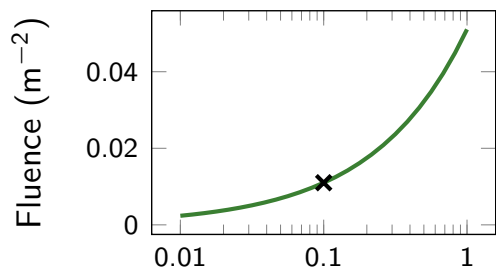

bulk density $(\mathrm{g} / \mathrm{cc})$

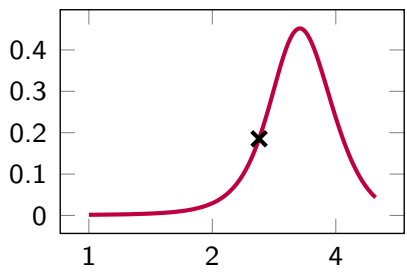

size index 


\section{Analytic model}

Fluence depends strongly on close approach distance

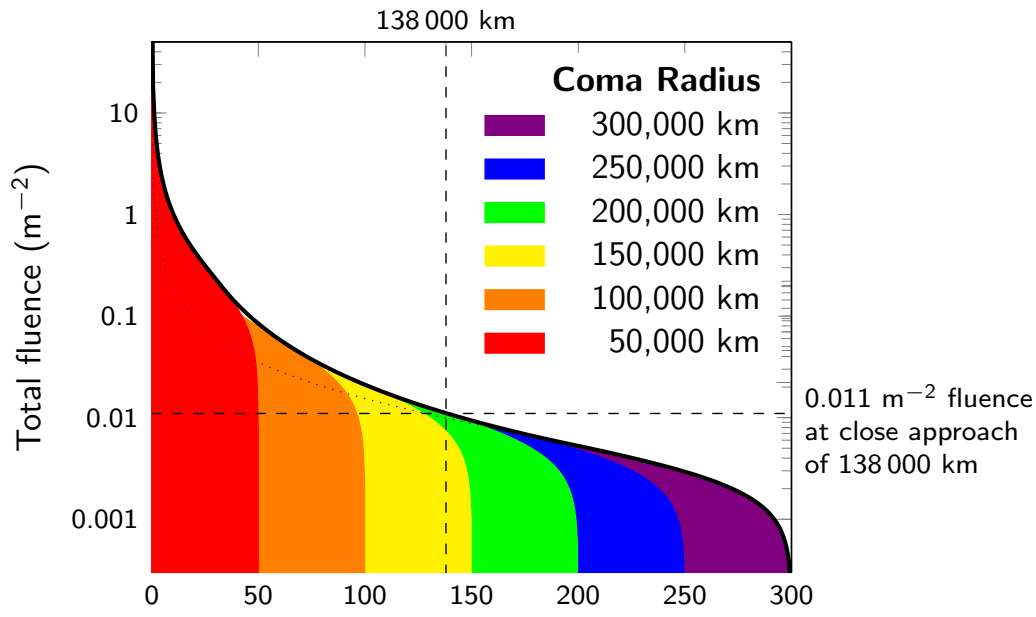

Close approach distance $\left(10^{3} \mathrm{~km}\right)$ 


\section{Validation \#1: reproducing Giotto results}

- We test our model by applying it to $1 \mathrm{P} /$ Halley

- Using a coma radius of $200,000 \mathrm{~km}$, we can reproduce the flux Giotto recorded

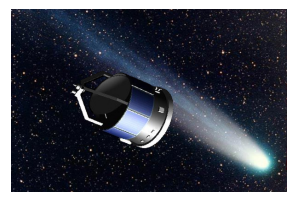

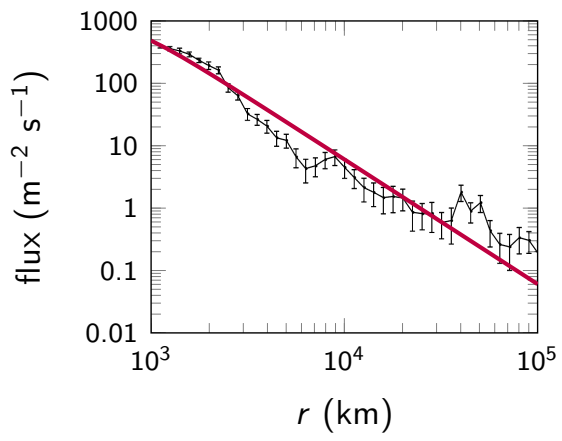

Fulle et al. (2000) data, our model 


\section{Validation \#2: reproducing Stardust results}

- Stardust flew 300 km from 81P/Wild 2

- Coma radius was $24,000 \mathrm{~km}$ at $1.7 \mathrm{au}$

- We model impacts per mass channel
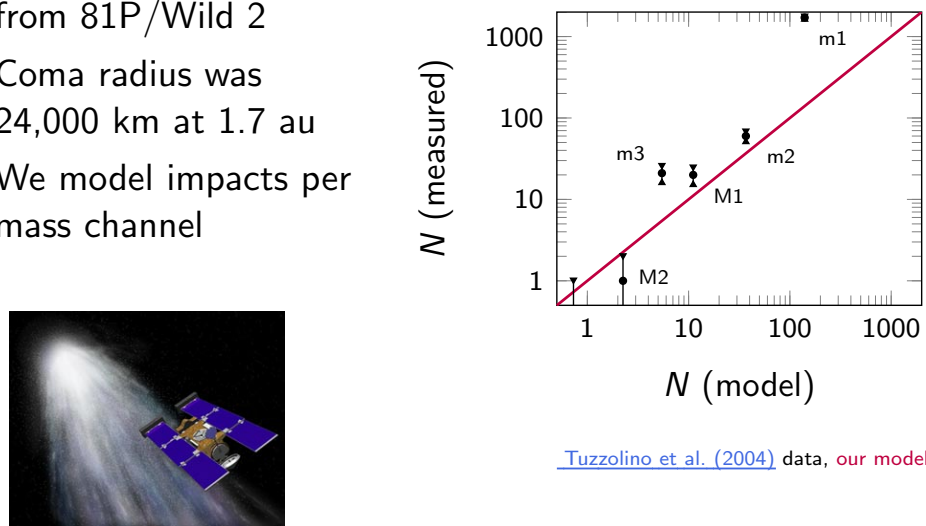

Tuzzolino et al. (2004) data, our model 


\section{Validation \#3: comparison with simulations $4 \times 10^{-6} \mathrm{~g}$ particles}

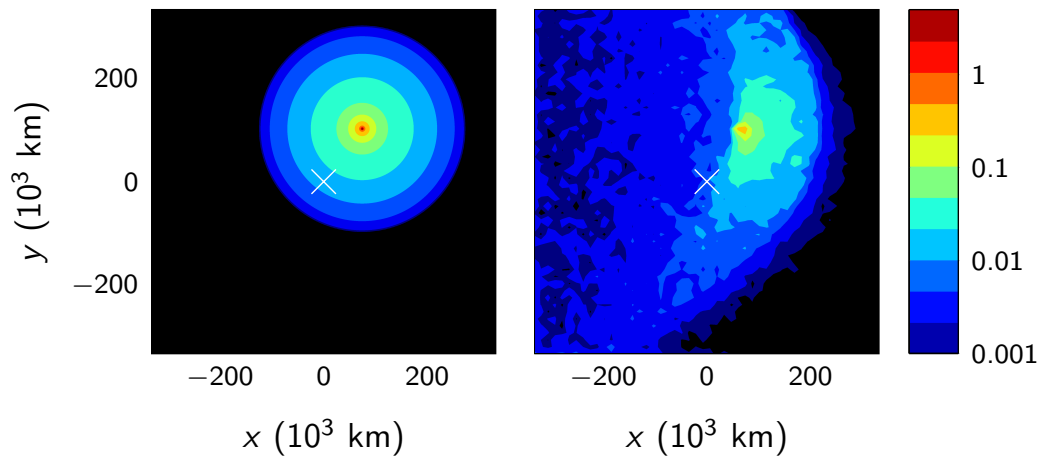

- Analytical model (left) and normalized simulations (right) in plane containing Mars (at origin), perpendicular to trajectory.

- Simulations performed by Paul Wiegert, UWO. 


\section{Validation \#3: comparison with simulations}

$4 \times 10^{-6} \mathrm{~g}$ particles

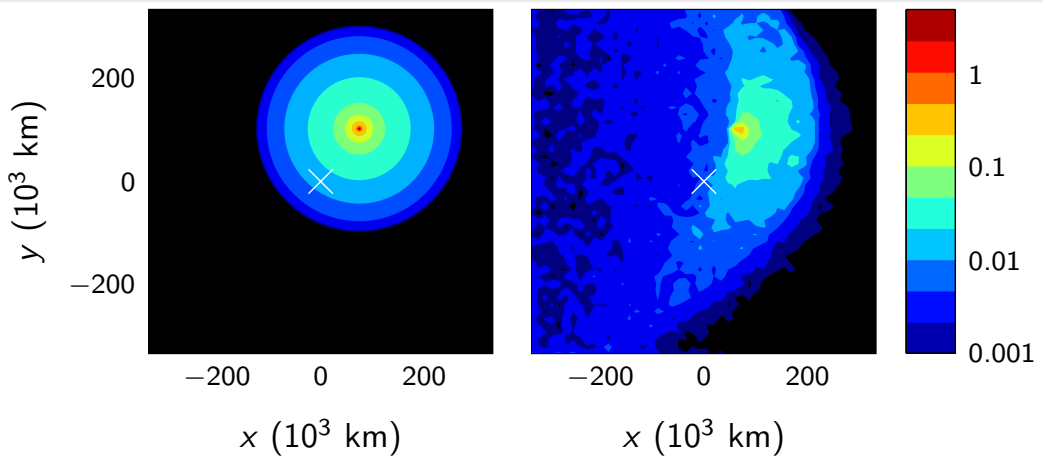

- Small scale simulations do not predict number of particles; fluence on right is multiplied by $N_{\text {theory }} / N_{\text {sim }}$.

- Simulations do illustrate (modest) deviance from spherical model due to coma asymmetry and tail. 


\section{Validation \#3: comparison with simulations $4 \times 10^{-6} \mathrm{~g}$ particles}

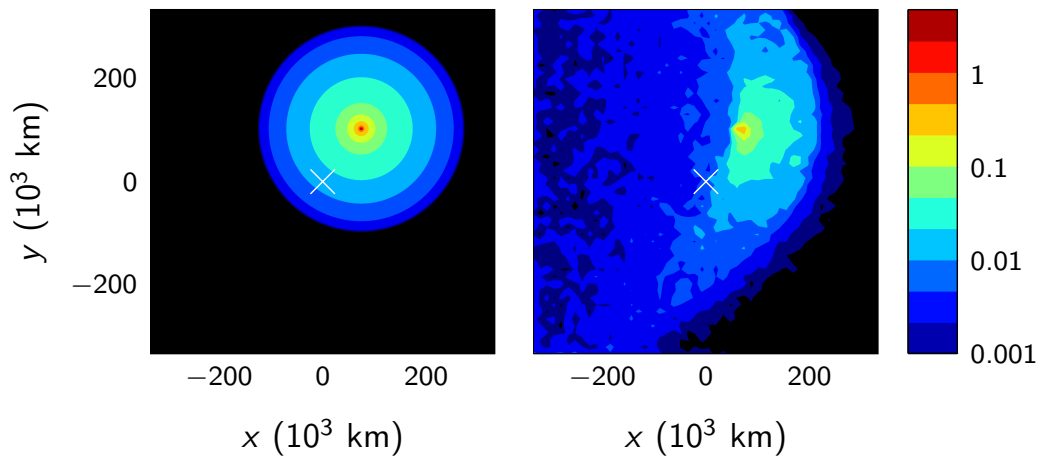

- Simulated coma has large radius $(200,000 \mathrm{~km})$ due to early assumed start of activity (10 AU)

- Effective radius may be smaller - needs further study 


\section{Validation \#3: comparison with simulations}

\section{$4 \times 10^{-3} \mathrm{~g}$ particles}

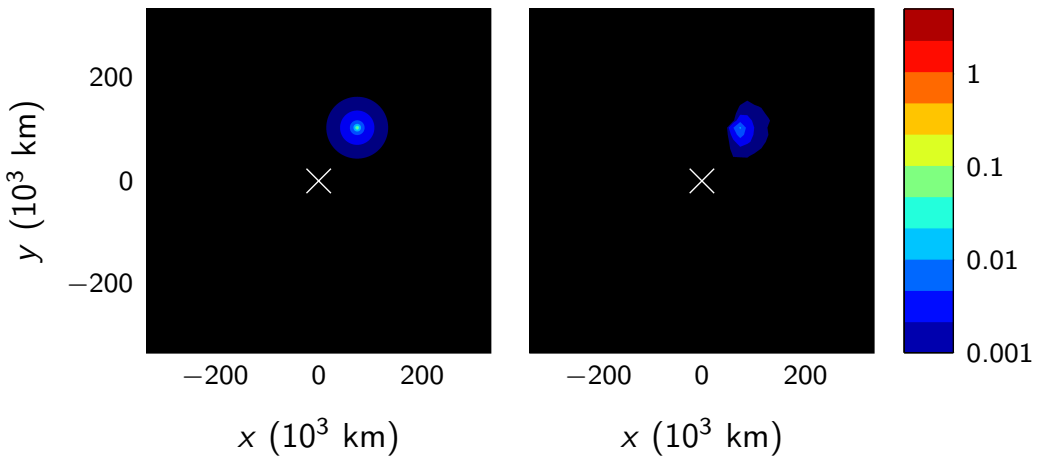

- Larger particles:

- Have lower ejection velocity

- Are less subject to radiative forces 


\section{Validation \#4: comparison with independent studies}

- Parallel effort to model coma particle dynamics: Vaubaillon et al., 2014

- Uses Af $\rho$ rather than magnitude to scale dust production.

- Results agree to within an order of magnitude ... with the same input parameters

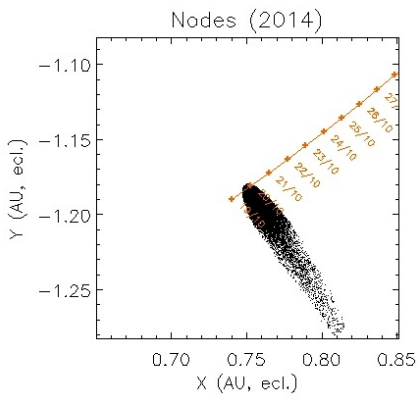




\section{Martian meteor shower}

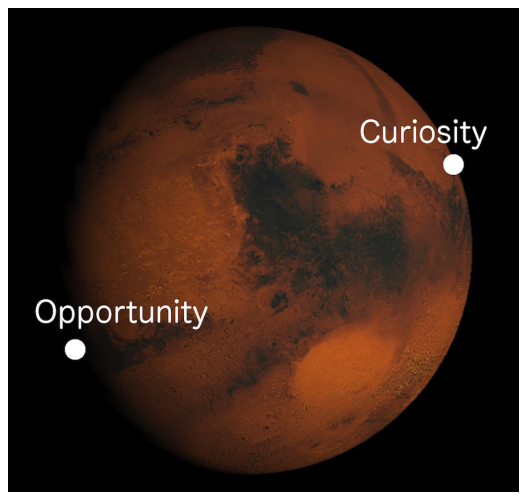

- Meteor shower will accompany Siding Spring

- ZHR for $10^{-6} \mathrm{~g}$ particles $\sim 30,000,000$ at Mars

- Subradiant near Opportunity at dawn

- MarsExpress may see up to 1000 meteors per minute

- $10,704 \mathrm{~km}^{2}$ FOV

- $m \gtrsim 3 \mathrm{~g}$

- Numbers from Anastasios Margonis 


\section{Meteoroid impact risks}

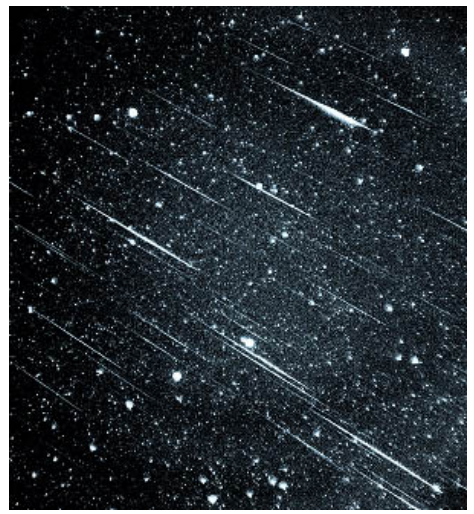

- Average flux of 100 micron or larger meteoroids in low Earth orbit is $5 \times 10^{-6} \mathrm{~m}^{-2}$ per hour

- Fluence due to Siding Spring is 500 times higher ( 5 years of LEO exposure)

- There has never been an event like this near Earth in recent memory, with the possible exception of the 1966 Leonids.

1966 Leonids by A. Scott Murrell 


\section{Effects on the Martian atmosphere}

- Atmospheric effects

- Energy deposition:

$$
\mathrm{KE}=\int \frac{d \sigma}{d m} m d m \times \frac{v^{2}}{2} \times \pi r_{M}^{2}
$$

- 1.14 megatons of TNT equivalent

- $0.006 \%$ of solar irradiance: insignificant

- Metal deposition probably more important (John Plane, Leeds) 


\section{Effects on the Martian system}

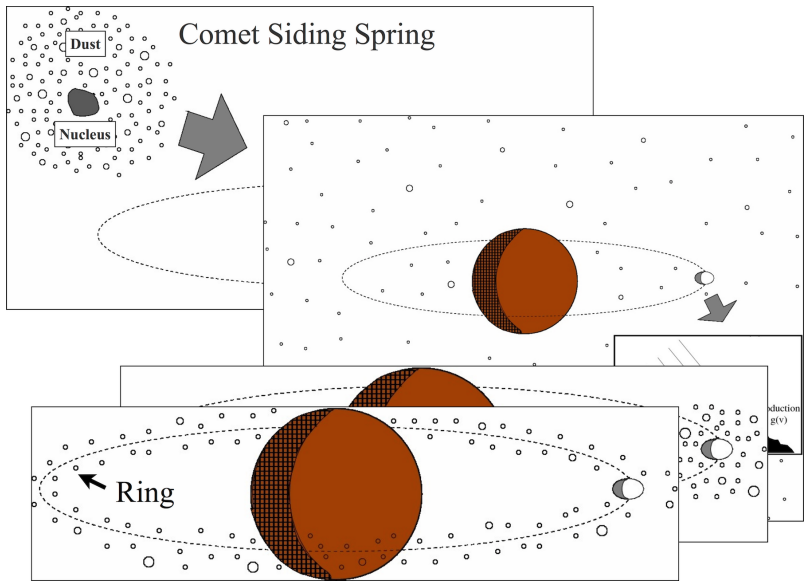

Impact ejecta from the moons could last months or years (Apostolos Christou, Armagh) 


\section{Brightness monitoring}

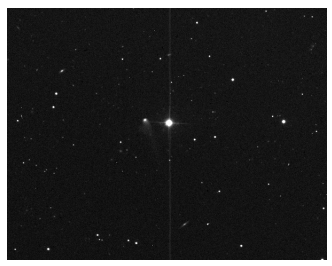

$1 \mathrm{~m}$ at Siding Spring

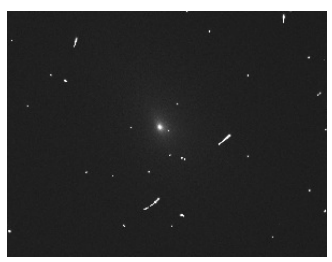

Hubble
Multiple efforts to monitor Siding Spring:

- NMSkies, 0.5m (MEO)

- Siding Spring, $1 \mathrm{~m}$ (MEO)

- Siding Spring, 2m (Christou, Armagh)

- NEOWISE

- Hubble (PI: Jian-Yang Li) 


\section{Brightness monitoring}

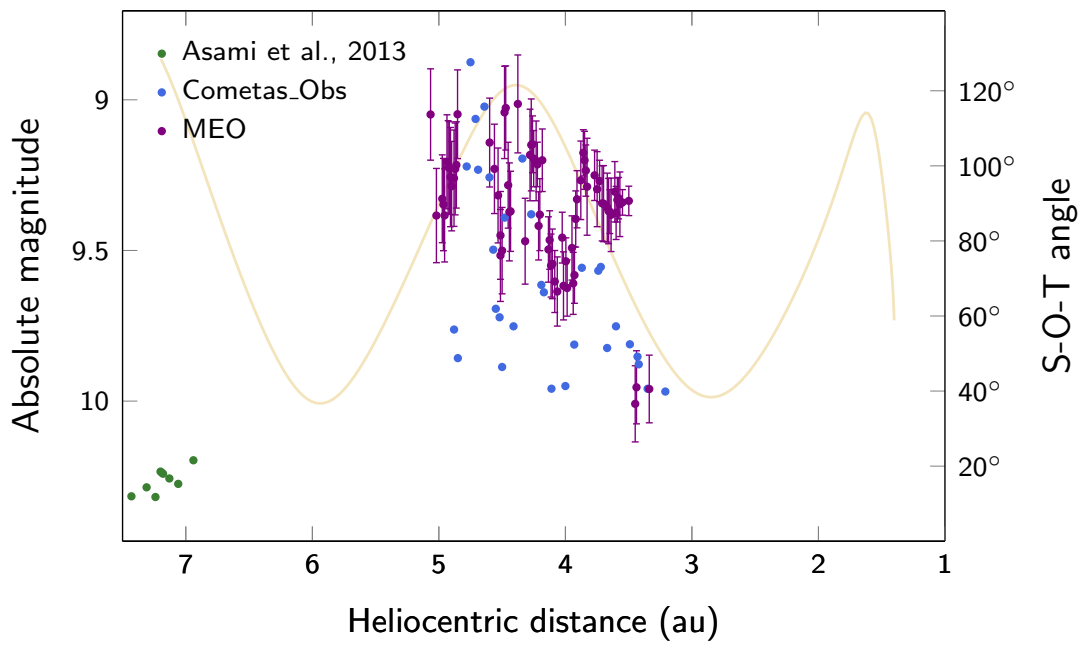




\section{Syndyne calculations}

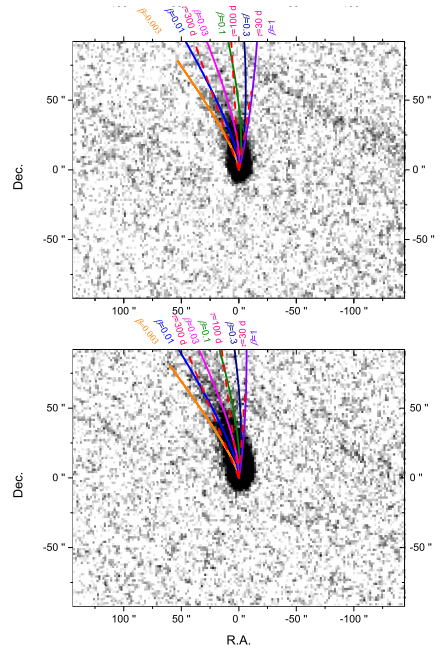

Ye \& Hui, 2014
- Ye \& Hui, 2014:

- Shape of cometary tail is influenced by gravity and radiation pressure

- "Mostly large particles" based on curvature of tail

- Low ejection velocity based on compactness of the coma

- Low ejection velocity

= compact coma

$=$ much lower fluence at Mars 


\section{Hubble observations}

\section{Comet Siding Spring • March 11, 2014 - Hubble Space Telescope • WFC3/UVIS}

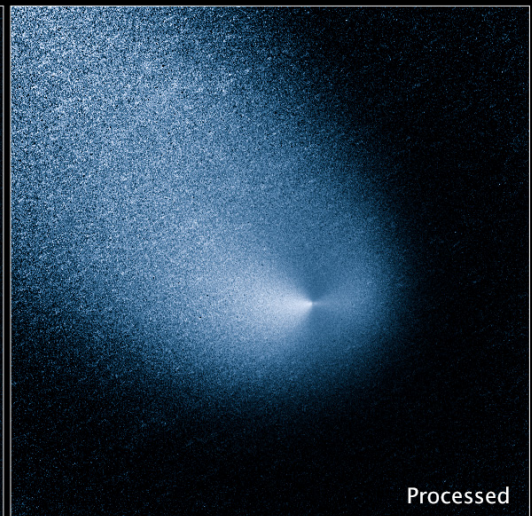




\section{Hubble observations}

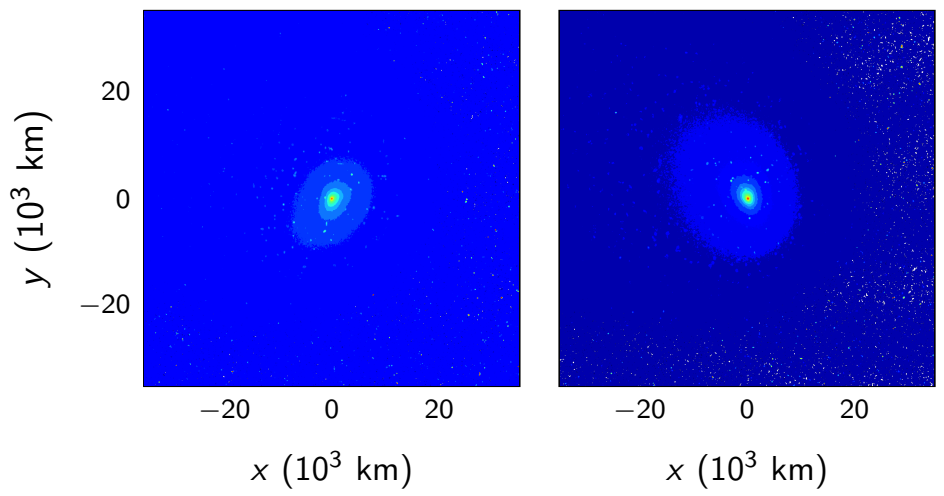

The coma does appear to be expanding slowly 


\section{Heliocentric magnitude: Hale-Bopp}

Hale-Bopp had three phases of activity pre-perihelion (Kidger, 1997)

$0 \propto r^{-5}$ at large distances

- $\propto r^{-1}$ around 4 au (a "standstill")

$\odot \propto r^{-3.5}$ at smaller heliocentric distances

Kidger notes that ice sublimation begins around 4 au 


\section{Heliocentric magnitude: Hale-Bopp}

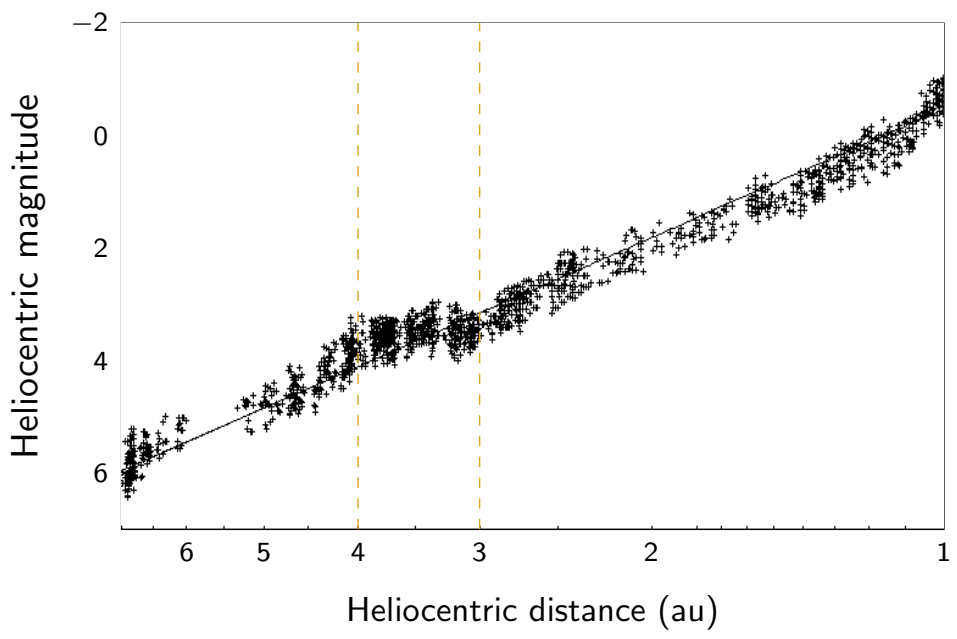

Image credit: A. Kammerer 


\section{Heliocentric magnitude: Siding Spring}

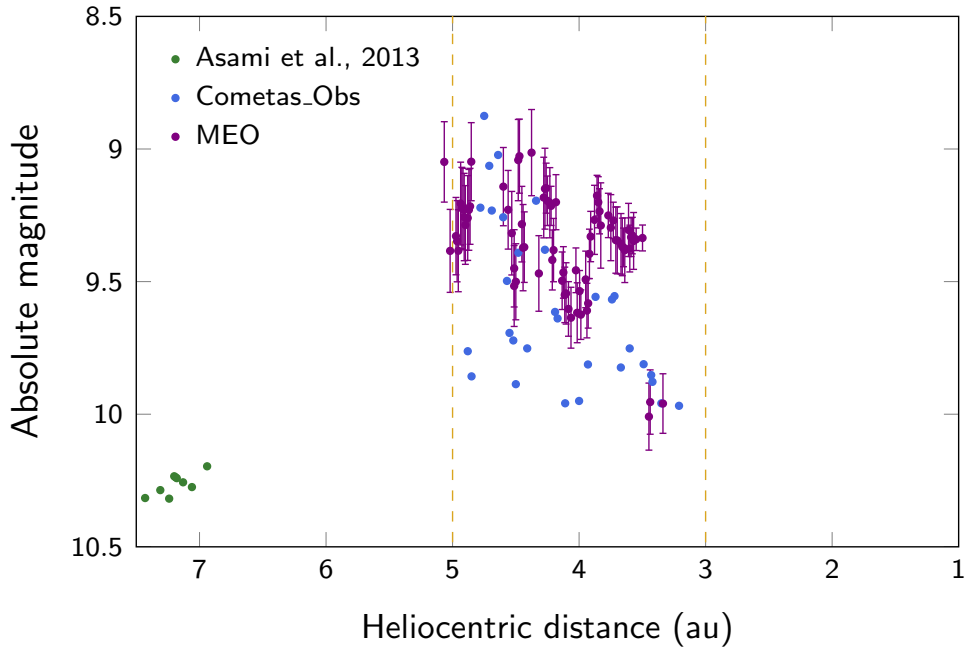




\section{Summary}

- Comet C/2013 A1 (Siding Spring) will have close encounter with Mars on October 19, 2014

- Mars and spacecraft will pass through coma and tail containing meteoroids

- Meteoroids $\left(4.19 \times 10^{-6} \mathrm{~g}\right.$ or larger $): \sim 1 \%$ chance of impact per square meter due to coma and tail

- Continued monitoring is needed to better predict future behavior 